\title{
EFFECT OF NOZZLES' CHARACTERISTICS ON PV AEROMECHANICAL SYSTEMS
}

\author{
LIRON SHANI ${ }^{1}$, RONEN S. LAUTMAN ${ }^{2}$ \& BOAZ NISHRI ${ }^{2}$ \\ ${ }^{1}$ Faculty of Engineering, Tel Aviv University, Israel \\ ${ }^{2}$ CoreFlow Ltd, Israel
}

\begin{abstract}
This paper presents results from a CFD analysis that highlights the effect of nozzles' characteristics on the performance of PV aeromechanical systems. PV aeromechanical systems enable accurate positioning of thin flexible substrate by creating an air cushion between the substrate and an accurate rigid surface, having bi-directional aeromechanical spring-like behavior. Nozzles can be described as the relation they allow between flow (Q) and pressure drop across them $(\Delta \mathrm{p}): \Delta \mathrm{p} \propto \mathrm{Qn}$ where $\mathrm{n}$ depends on the characteristic behavior and (in this work) is between 1 and 2 . The characteristic behavior depends on the mechanism by which pressure is reduced. The mechanism can be dominated by inertial effects, by viscous effects, or by a combination of both inertial and viscous effects. It was found that aeromechanical performance is very sensitive to the nozzles' characteristic. An air cushion with high aeromechanical stiffness and constant flow rate is achieved by combining vacuum nozzles of exponent $\mathrm{n}=1$ and pressure nozzles of exponent $\mathrm{n}=2$.
\end{abstract}

Keywords: CFD, air floating, non-contact handling, aeromechanical spring.

\section{INTRODUCTION}

This paper highlights a new understanding of fluid dynamics of an accepted technique for accurate positioning and maintaining thin flexible substrate positioning over a surface where non-contact with the substrate is beneficial. There are a large variety of applications, such as manufacturing LCD or OLED screens where non-contact, accurate handling of thin glass is required. Fabrication processes such as surface inspection, cleaning, coating, heating, and etching are good examples of these. A common solution for maintaining thin flexible substrate non-contact accurate positioning is to use a Pressure to Vacuum (PV) aeromechanical support system.

PV aeromechanical systems include a rigid surface with periodic distribution of openings which supply either pressure or vacuum, as well as appropriate connections to pressure and vacuum supplies (typically air or $\mathrm{N}_{2}$ gas). A thin substrate covering the rigid surface will experience the pressure as a force directed away from the surface, and the vacuum as a force directed toward the surface. The distance between the substrate and rigid surface is called an air gap or air cushion. The pressure field in the air gap is determined by the pressure/vacuum supplies, the air gap height, and the nozzles which are located between the pressure/vacuum supplies and the air gap. These nozzles require a significant pressure drop to allow flow through them, thus allowing the net force on the substrate to be zero at the non-zero equilibrium air gap. At an air gap smaller than equilibrium the net force acting on the substrate is directed away from the surface; at an air gap larger than equilibrium the net force acting on the substrate is directed toward the surface. The changing air gap height requires a change in flowrate. The changing flowrate through each nozzle requires a change in the pressure drop across each nozzle. Thus, the nozzles allow a uniform, constant pressure supply to result in a differing pressure field on a substrate, which will be a net force on the substrate in the direction toward equilibrium. This relationship between air gap height and force can be quantified into a bi-directional aeromechanical spring-like stiffness, as shown in [1]. 
Air cushion stiffness is the most significant performance parameter of PV aeromechanical systems. It determines both the static performance, and dynamic performance. To take two examples from the field of Flat-Panel Display (FPD), static performance is required in inspection applications which use "step-and-scan" methodologies, and dynamic performance is required in coating applications where a substrate moves over a PV platform while coating material is being deposited with a tight tolerance.

Low-stiffness PV aeromechanical systems may provide positioning accuracy of $\pm 100 \mu \mathrm{m}$ and stability of approximately $10 \mu \mathrm{m}$. By contrast, a high-stiffness PV system may provide positioning accuracy of $\pm 1 \mu \mathrm{m}$ and stability of approximately $0.02 \mu \mathrm{m}$.

Design of stiff air cushions involves multiple parameters and the interaction between them. For example, the flow circuits need to be designed with suitable ratio between the pressure and vacuum nozzles resistance $R_{p}$ and $R_{v}$, respectively. As another example, increasing the flow rate will increase the aerodynamic forces involved as well as the stiffness. The implication of high flow is high power consumption and difficulty to distribute the flow evenly across the all PV surface. Uniform air distribution across the platform is critical to the uniformity of aeromechanical performance. It is therefore valuable to optimized the PV aeromechanical system in terms of the aerodynamic parameters without arbitrarily large flowrates.

The PV aeromechanical system performance is governed by the reaction of the vacuum and pressure nozzles to changing flow rate. The relationship between pressure drop and flowrate includes both viscous and inertial terms. Dominance of either the viscous or the inertial term will determine the nozzle behavior, which can be characterized by the DarcyForchheimer relation (eqn (1)) that was originally formulated for flows in porous media permeability, for example in [2]

$$
-\frac{\Delta P}{L}=\alpha \mu V+\beta \rho V^{2}
$$

where $\Delta P / L$ is pressure drop per length, $\mu$ is gas viscosity, $V$ is average gas velocity, $\rho$ is gas density, $\alpha$ and $\beta$ are two constants: $\alpha$ related to the viscous term and $\beta$ to the inertial term. To describe PV nozzles behavior, Darcy-Forchheimer may simplified to a relation between flow rate $(Q)$ and pressure drop $(\Delta P)($ eqn $(2))$. $k$ is about constant for small $\Delta P$

$$
-\frac{\Delta P}{L}=k Q^{n}
$$

Previous work was done to try to predict the viscous effects of flow through a duct of variable cross-section by Armengol et al. in [3], which helps inform the behavior of airflow in a PV aeromechanical system. As the air flows from the pressure opening, it must flow outward in an almost axisymmetric fashion toward all four neighbouring vacuum openings. The current work presents Computational Fluid Dynamics (CFD) results on PV aeromechanical platforms. The main goal is to find the effects that nozzles' characteristics have on the air gap stiffness at constant flow rate. Nozzles' characteristics is defined by the exponent $n$ in eqn (2), where $n$ is between 1 and 2, and by the constant $k$. In the discussion below np and $k_{p}$ refer to the exponent and constant relating to the pressure nozzle, respectively. nv and $\mathrm{k}_{\mathrm{v}}$ refer to the exponent and constant relating to the vacuum nozzle, respectively.

\section{CFD MODEL}

Finite-volume based CFD software "Fluent" was used to analyse the effect of nozzles' characteristics on PV aeromechanical stiffness in [4]. The analysis domain is presented in Fig. 1. The distance $d$ between pressure and vacuum openings is selected to be small enough 


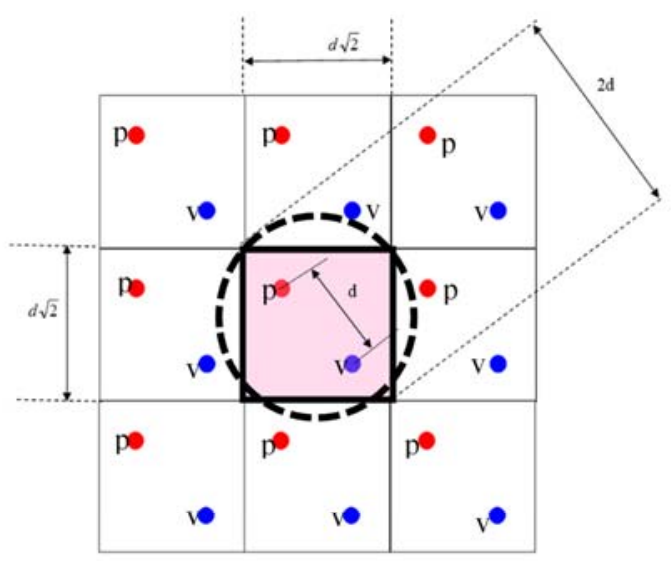

(a)

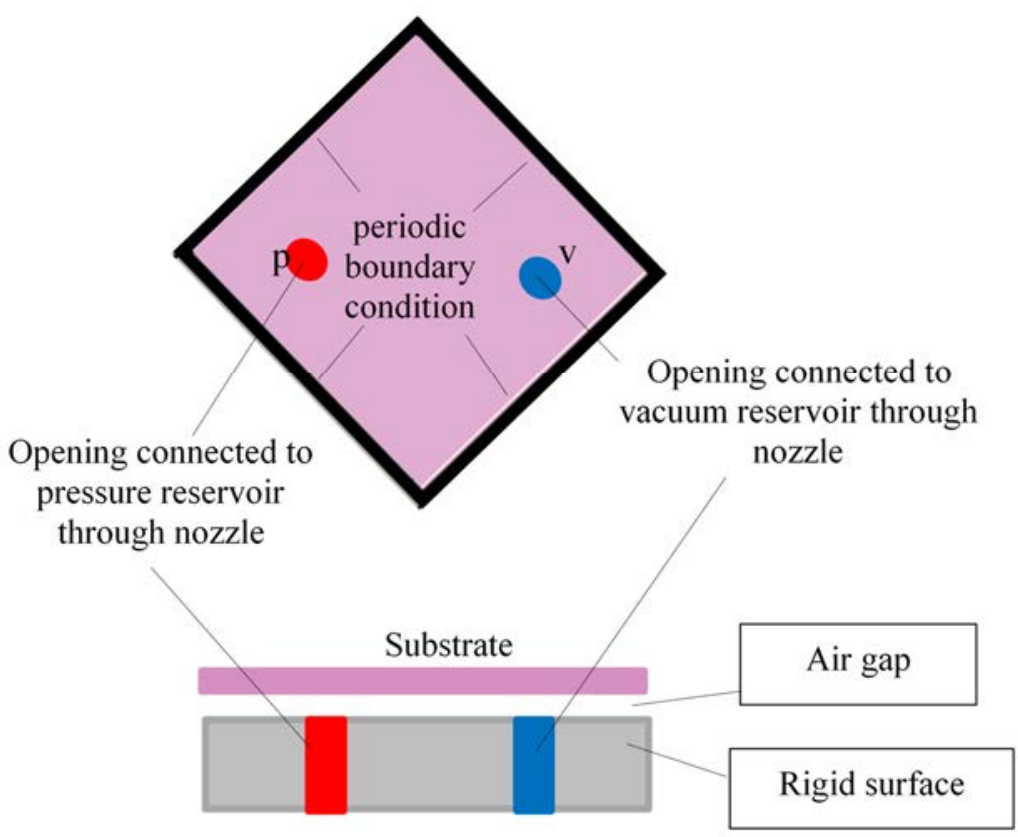

(b)

Figure 1: (a) Outline of infinite distribution of pressure and vacuum openings in a rigid surface. The distance between neighbouring openings is $\mathrm{d}$. The repeating nature of the distribution allows analysis of only a one basic cell with periodic boundary conditions; (b) Top and side views of the basic cell structure of the CFD analysis domain. The inlet is the opening for a pressure nozzle, which is simulated as a reservoir at constant pressure. The outlet is the opening for a vacuum nozzle, which is simulated as a reservoir at constant vacuum. The air gap is of constant height. The substrate and rigid surface are modelled as non-slip walls. The boundaries in the four other sides are modelled as periodic. 
that substrate deformation due to local pressure extrema is very small. As a result, the basic cell pressure-vacuum field can be represented by single net force: the pressure-vacuum field integration over the basic cell.

The CFD analysis domain encloses one pressure opening and one vacuum opening. The boundaries of the analysis domain as the rigid surface and the thin substrate surface (impermeable walls). The analysis domain approximates an infinitely large rigid surface, and so there is an explicit assumption made that the flow between the pressure and vacuum openings are periodic in the two directions normal to the air gap height. The periodic boundaries are shown in thick black lines in Fig. 1(b).

The inlet to the analysis domain is simulating a reservoir at constant pressure, and the outlet is simulating a reservoir at constant vacuum. Inside of the opening of the pressure nozzle is a volume of calculation cells which are governed by the characteristic behavior of the pressure nozzle, $\Delta \mathrm{P}=\mathrm{k}_{\mathrm{p}}{ }^{*} \mathrm{Q}^{\mathrm{np}}$. Inside the opening of the vacuum nozzle is a volume of calculation cells which are governed by the characteristic behavior of the vacuum nozzle, $\Delta \mathrm{P}=\mathrm{k}_{\mathrm{v}} * \mathrm{Q}^{\mathrm{nv}}$.

The numerical mesh was a structured grid of hexahedrons. The mesh was defined very finely in the vicinity of the pressure and vacuum openings, and more coarsely near the periodic boundaries where the flow was expected to change slower. There were 714,812 elements in the model whose solution was found to be grid independent.

Fig. 2(a) shows the net force over the PV cell as function of air gap height, as well as the stiffness (which is the derivative of the force) as a function of the air gap height. The graph of the force shows that when the gap height deviates from the equilibrium air gap in either direction then the direction of the net force acts in the direction back toward equilibrium. The aeromechanical system provides stable equilibrium. The relevant practical stiffness is at the equilibrium air gap, which is $50 \mu \mathrm{m}$ in this example. Fig. 2(b) shows a typical pressure field distribution over a PV basic cell at equilibrium.

\section{RESULTS}

Fig. 3 shows the stiffness of five different PV air gap designs, all of which have an equilibrium air gap of $50 \mu \mathrm{m}$. The difference between the five designs is in the characteristic behavior of the nozzles. In each case the pressure and vacuum nozzles have the same characteristic, as shown in the legend. The flow rate, pressure supply, and vacuum supply are identical for all cases. The air gap with both nozzles' characteristic $n=2$ provides $20 \%$ more stiffness than the air gap with both nozzles' characteristic $n=1$.

Fig. 4 shows the stiffness of different PV air gap designs, all of which have an equilibrium air gap of $50 \mu \mathrm{m}$. The difference between the five designs is in the characteristic behavior of the nozzles. In each case the pressure and vacuum nozzles have different characteristics, as shown in each graph title and legend. For any pressure nozzle characteristic $n p=$ constant, vacuum nozzle characteristic $n v=1$ provides higher stiffness compared with $2 \geq n v>1$.

Fig. 5 presents the relative stiffness at nominal gap for all combinations of air gap designs within the range of $n p=1$ to 2 and $n v=1$ to 2 . To clarify, this figure does not show the stiffness of the air gap as it deviates from equilibrium. Relative stiffness $=100 \%$ was arbitrarily selected as the average stiffness in the range. The flow rate, pressure supply, and vacuum supply are identical for all cases. Minimum stiffness is observed at the combination of $n p=1$ and $n v=2$. Maximum stiffness, more than $100 \%$ higher than the minimum, is observed at the combination of $n p=2$ and $n v=1$. 
(a)

(b)
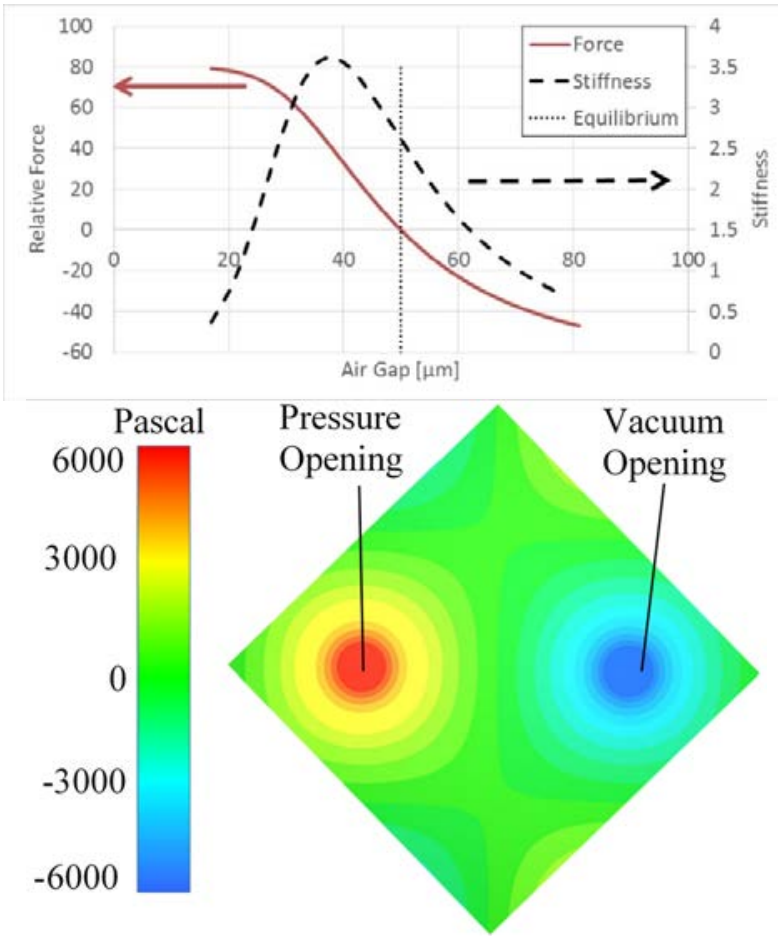

Figure 2: (a) Net force over the PV cell and its derivative (stiffness) as function of air gap height. Positive force is in the direction away from the rigid surface; (b) A typical pressure field distribution over a PV basic cell at equilibrium.

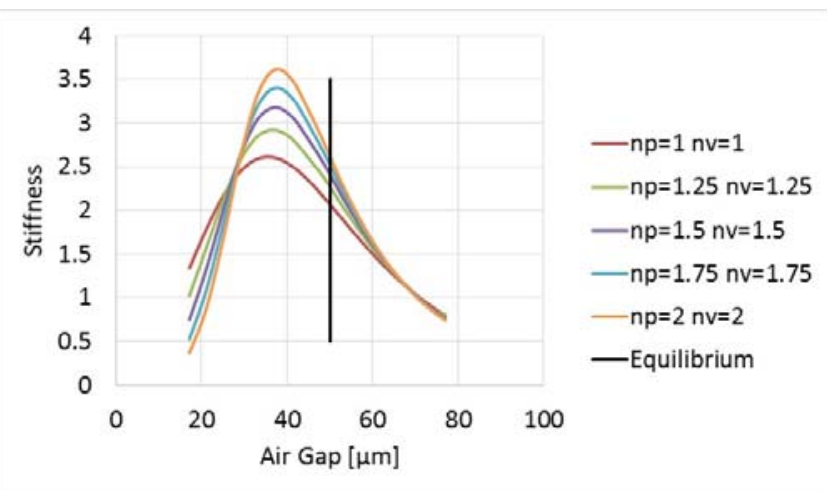

Figure 3: Stiffness of five different PV air gap designs, all of which have an equilibrium air gap of $50 \mu \mathrm{m}$. The difference between the five designs is in the characteristic behavior of the nozzles. In each case the pressure and vacuum nozzles have the same characteristic, as shown in the legend. The flow rate, pressure supply, and vacuum supply are identical for all cases. The air gap with both nozzles' characteristic $\mathrm{n}=2$ provides $20 \%$ more stiffness than the air gap with both nozzles' characteristic $n=1$. 


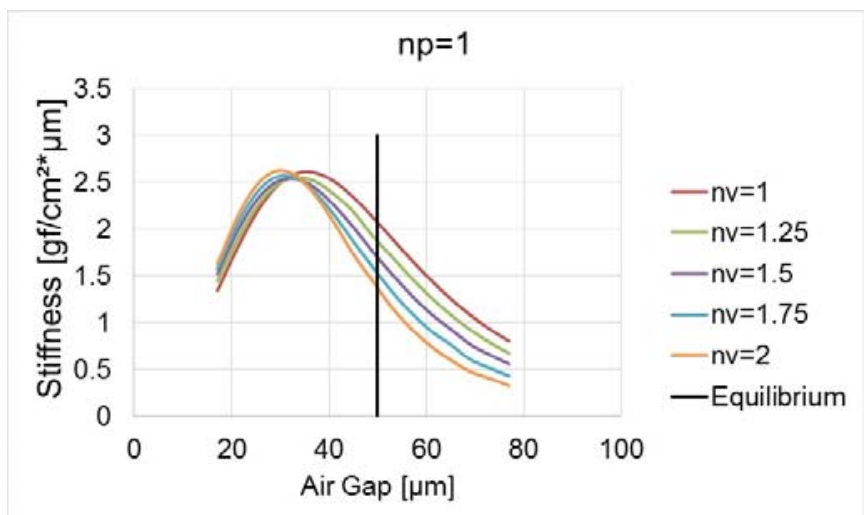

(a)

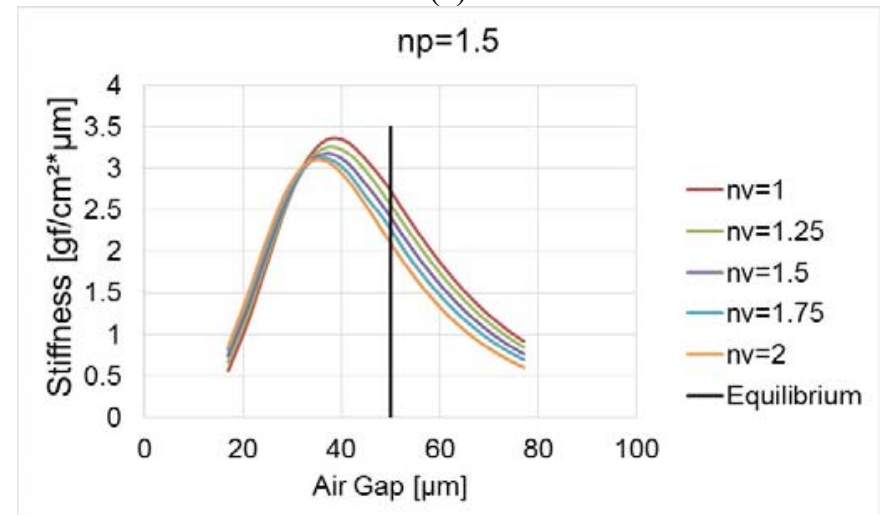

(b)

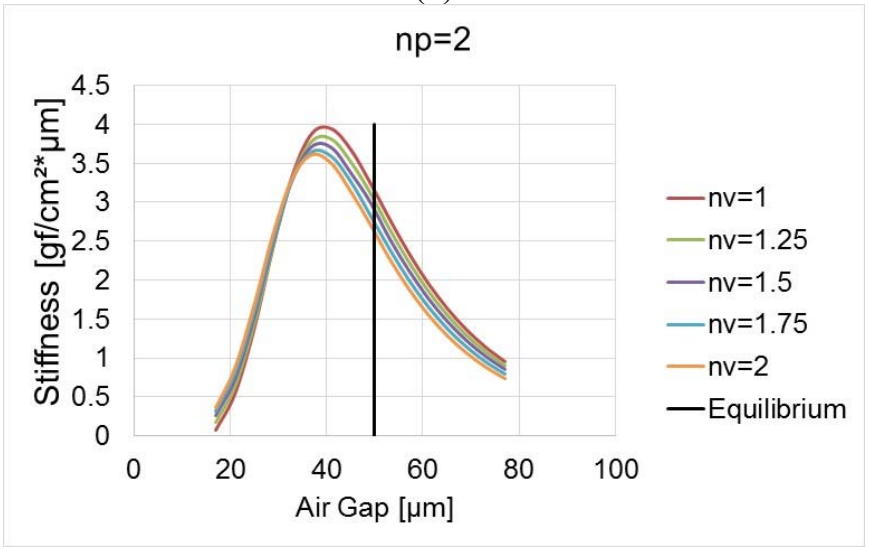

(c)

Figure 4: Stiffness of different PV air gap designs, all of which have an equilibrium air gap of $50 \mu \mathrm{m}$. The difference between the five designs is in the characteristic behavior of the nozzles. In each case the pressure and vacuum nozzles have different characteristics, as shown in each graph title and legend. For any pressure nozzle characteristic $n p=$ constant, vacuum nozzle characteristic $n v=1$ provides higher stiffness compared with $2 \geq n v>1$. 


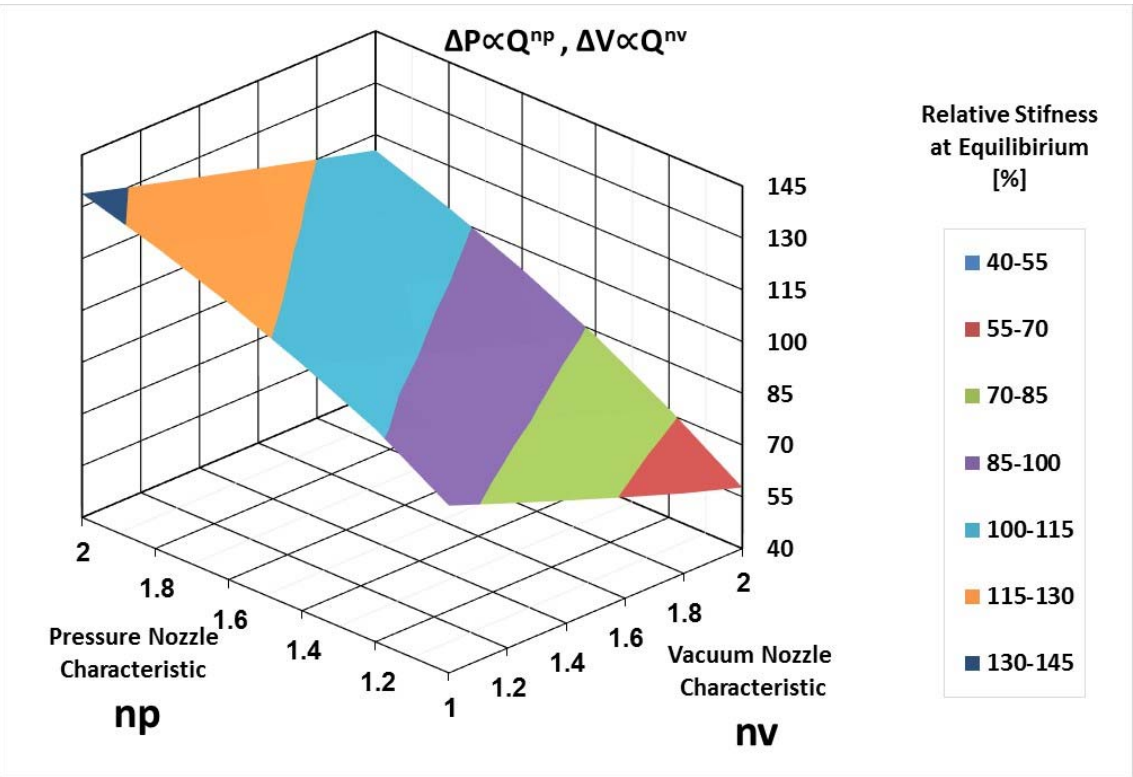

Figure 5: Relative stiffness at nominal gap for all combinations of air gap designs within the range of $n p=1$ to 2 and $n v=1$ to 2 . Relative stiffness $=100 \%$ was arbitrarily selected as the average stiffness in the range. The flow rate, pressure supply, and vacuum supply are identical for all cases. Minimum stiffness is observed at the combination of $n p=1$ and $n v=2$. Maximum stiffness, more than $100 \%$ higher than the minimum, is observed at the combination of $n p=2$ and $n v=1$.

\section{DISCUSSION AND SUMMARY}

This paper presents the results of CFD analyses that highlight the nozzles' characteristics effect on the performance of a PV aeromechanical system. It was found that the nozzles' characteristics play a major role on PV performance. Efficient PV with high stiffness is achieved by combination of low characteristic exponent for vacuum nozzles and high characteristic exponent for pressure nozzles. The characteristic exponent for each nozzle informs the selection of nozzle types when designing a new system. Vacuum nozzles should be designed for viscous effects to dominate the behavior, and pressure nozzles should be designed for inertial effects to dominate the behavior.

\section{REFERENCES}

[1] Yassour, Y., Richman, H. \& Levin, D., High-Performance Non-Contact Support Platforms. US Patent 7530778B2, 2009.

[2] Andrade, J.S. et al., Inertial effect on fluid flow through disordered porous media. Physical Review Letters, 82(26), pp. 5249-5252, 1999.

[3] Armengol, J. et al., Bernoulli correction to viscous losses: radial flow between two parallel discs. American Journal of Physics, 76, pp. 730-737, 2008.

[4] Shani, L., Nozzles characteristic effect on PV air cushion performance study. MSc thesis, Faculty of Engineering, Tel Aviv University, 2015. 